\title{
Performance Evaluation and Comparison of Fuzzy-based Intelligent CAC Systems for Wireless Cellular Networks
}

\author{
Leonard Barolli*, Gjergji Mino ${ }^{\dagger}$, Taku Ikebata ${ }^{\dagger}$, Admir Barolli ${ }^{\ddagger}$, Fatos Xhafa ${ }^{\S}$ Makoto Takizawa $^{\ddagger}$ \\ * Department of Information and Communication Engineering \\ Fukuoka Institute of Technology (FIT) \\ 3-30-1 Wajiro-Higashi, Higashi-Ku, Fukuoka 811-0295, Japan \\ E-mail: barolli@fit.ac.jp \\ $\dagger_{\text {Graduate School of Engineering }}$ \\ Fukuoka Institute of Technology (FIT) \\ 3-30-1 Wajiro-Higashi, Higashi-Ku, Fukuoka 811-0295, Japan \\ E-mail: gjmino@gmail.com,mgm10002@bene.fit.ac.jp \\ ${ }^{\ddagger}$ Department of Computers and Information Science, \\ Seikei University, \\ 3-3-1 Kichijoji-Kitamachi, Musashino-Shi, Tokyo 180-8633, Japan \\ E-mail: admir.barolli@gmail.com,makoto.takizawa@computer.org \\ $\S$ Department of Languages and Informatics Systems \\ Technical University of Catalonia \\ Jordi Girona 1-3, 08034 Barcelona, Spain \\ E-mail: fatos@lsi.upc.edu
}

\begin{abstract}
The mobile cellular systems are expected to support multiple services with guaranteed Quality of Service (QoS). But, the ability of wireless systems to accommodate expected growth of traffic load and broadband services is limited by available radio frequency spectrum. Call Admission Control (CAC) is one of the resource management functions, which regulates network access to ensure QoS provisioning. However, the decision for $\mathrm{CAC}$ is very challenging issue due to user mobility, limited radio spectrum, and multimedia traffic characteristics. To deal with these problems, we implemented a Fuzzy Admission Control System (FACS). We compared the performance of FACS with Shadow Cluster Concept (SCC). In another work, we extended FACS by considering the priority of the on-going connections. We called this system FACS-P. As priority parameter, we considered only one parameter (service request). In this work, we improve our previous system by adding different priorities. We call this system FACS-MP. We evaluate and compare the performance of implemented systems by simulations. From the simulations results, we conclude that the FACS-MP can differentiate better different services compared with previous systems.
\end{abstract}

Keywords-Wireless Cellular Networks; CAC; Fuzzy Logic; Priorities.

\section{INTRODUCTION}

As the demand for multimedia services over the air has been steadily increasing over the last few years, wireless multimedia networks have been a very active research area [1]. To support various integrated services with a certain Quality of Service (QoS) requirement in these wireless networks, resource provisioning is a major issue.
To guarantee the QoS, Call Admission Control (CAC) is a good strategy. CAC is a provisioning strategy that limits the number of connections into the network in order to reduce the network congestion and call dropping.

$\mathrm{CAC}$ is not a problem that is unique to wireless networks. It is applicable to almost every type of networks, but in cellular wireless networks due to users' mobility the CAC becomes much more complicated. While in wired networks the resources are reserved for the call at set-up time and are not changed after that, in cellular wireless networks when the mobile node moves from one cell to another one, the bandwidth must be requested in the new cell. During this process, the call may not be able to get a channel in the new cell due to the limited resource in wireless networks, which will lead to the call dropping. Thus, the new and handoff calls have to be treated differently in terms of resource allocation. Since users are more sensitive to call dropping than to call blocking, the hand-off calls are assigned higher priority than new calls [2].

CAC techniques are required to guarantee QoS requirements for all traffic types. In order to improve the system performance at the call level, a CAC strategy may block additional calls even if there are enough resources for their service. CAC is based on the knowledge of the statistical characteristics of ongoing and arriving calls [2]. The decision to accept an additional call involves the calculation or estimation of the consequences of the call acceptance on blocking and delay of itself and other incoming calls [3]. 
Several schemes have been proposed for CAC in wireless cellular networks. However, during the complexity of CAC in wireless environment, many simplified models and assumptions are made. Some schemes consider that each mobile node will make hand-over to neighbouring cells with equal probability, which may be not accurate in general. For this reason, the intelligent and heuristic methods are needed.

Use of intelligent methods based on Fuzzy Logic (FL), Neural Networks (NN) and Genetic Algorithms (GA) can prove to be efficient for traffic control in telecommunication networks [4-13].

In $[14,15]$, in order to deal with CAC in wireless cellular networks, we proposed a CAC system based on FL. We called this system FACS. We implemented and evaluated the proposed system by comparing its performance with Shadow Cluster Concept (SCC) [16].

In $[17,18]$, we proposed another FL-based CAC system by considering the priority of the on-going connections. However, as priority parameter, we considered only one parameter (service request). We called this system FACSP.

In this work, we extend our previous work by adding different priorities. We evaluate by simulations the proposed system and compare its performance with our previous work.

The structure of this paper is as follows. In Section II, we present the previous work. In Section III, we introduce the proposed system. In Section IV, we discuss the simulation results. Finally, some conclusions are given in Section V.

\section{Previous Work}

\section{A. $S C C$}

One of the previous work on CAC is SCC [16]. The fundamental idea of the SCC is that every mobile terminal with an active wireless connection exerts an influence upon the cells and their Base Stations (BSs) in the vicinity of its current location and along its direction of travel. As an active mobile terminal travels to other cells, the region of influence also moves, following the active mobile terminal to its new location. The BSs (and their cells) currently being influenced are said to form a shadow cluster, because the region of influence follows the movements of the active mobile terminal like a shadow, as shown in Fig. 1.

The shadow (and therefore the level of influence) is strongest near the active mobile terminal, and fades away depending on factors such as the distance to the mobile terminal, current call holding time and priority, bandwidth resources being used, and the mobile terminal's trajectory and velocity. Because of these factors, the shape of a shadow cluster is usually not circular and can change over time. The center of a shadow cluster is not the geometric center of the area described by the shadow, but the cell where the mobile terminal is currently located. This cell is considered as the mobile terminal's current home cell. A bordering neighbour is a cell that shares a common border with the shadow cluster's center cell. In contrast, a non-bordering neighbour cell, although being a part of the shadow cluster, does not share a border with the shadow cluster's center cell.

Conceptually, the number and "darkness" of the shadows covering a cell reflect the amount of resources that the cell's BS needs to reserve in order to support the active mobile terminals currently in its own and in neighbouring cells. With the information provided by shadow clusters, BSs can determine, for each new call request, whether the request can be supported by the wireless network. In practice, a shadow cluster is a virtual message system where BSs share probabilistic information with their neighbours on the likelihood that their active mobile terminals will move to neighbour cells (while remaining active) in the near future. With the information provided by shadow clusters, BSs project future demands and reserve resources accordingly. BSs reserve resources by denying network access to new call requests, and by "waiting" for active users to end their calls.

The decision process for the acceptance of a new call request also involves a shadow cluster. Every new call request results in the implementation of a tentative shadow cluster. BSs exchange information on their new call requests, and decide, based on this and other information, which requests should be accepted and which requests should be denied.

After a hand-off, BSs within the old shadow cluster are notified about this movement, and the mobile terminal's new current BS has to assume the responsibility of supplying the appropriate information to the BSs within the new shadow cluster. BSs which were in an old shadow cluster that has just moved away must delete any entries corresponding to the active mobile terminal that established the shadow cluster, and free reserved resources if appropriate. BSs which become part of the influence region of a shadow cluster must be given appropriate information on the shadow cluster's active mobile terminal, such as the respective QoS requirements, e.g., bandwidth demands, call dropping probabilities, and any other useful information such as the wireless connection's elapsed time, for the establishment of the new shadow cluster.

\section{B. FL-based CAC Systems}

In $[14,15]$, in order to deal with CAC in wireless cellular networks, we proposed a CAC scheme based on FL.

Conventional CAC schemes for wireless networks must consider some measured parameters to make the decision. However, in wireless networks due to the user mobility and varying of channel condition the measurement obtained are not accurate. Also, it is very difficult to obtain the complete statistics of the input traffic. Therefore, the CAC decision must be made based on the uncertain or inaccurate information. This is why we use FL. 


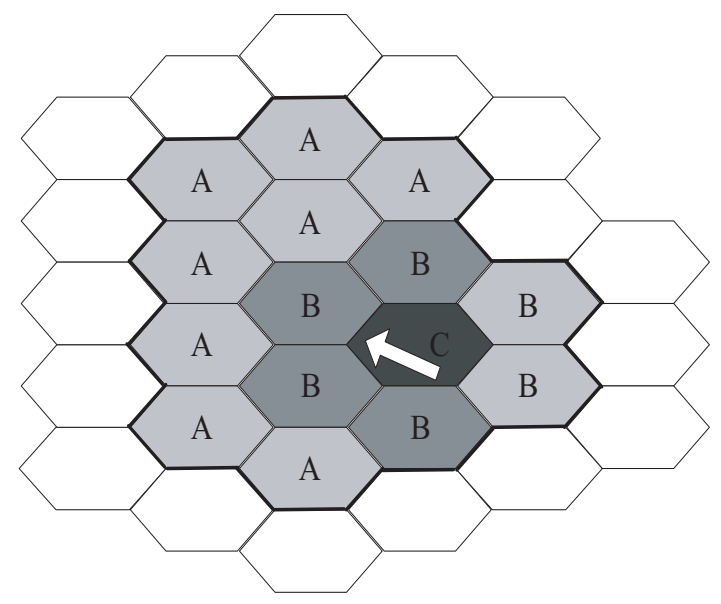

Figure 1. SCC.

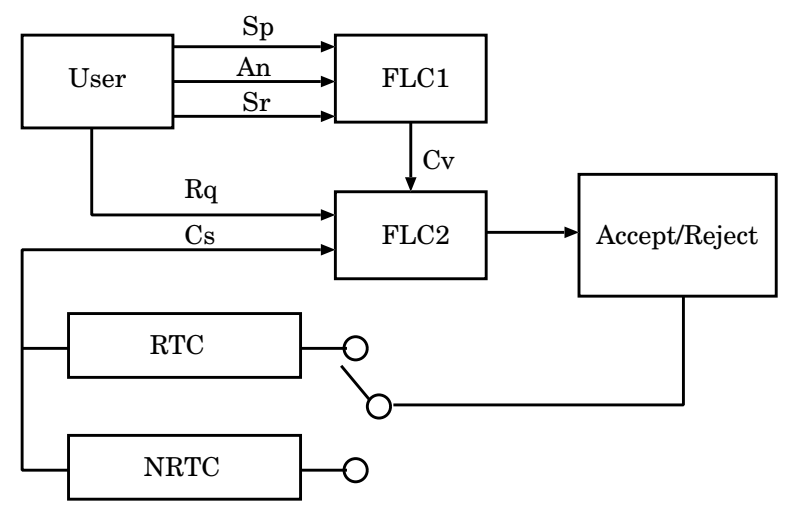

Figure 2. FACS-P model.

We implemented and evaluated the proposed system by comparing its performance with SCC [16]. We showed that the proposed scheme could achieve a better prediction of the user behaviour and a good admission decision compared with SCC.

In $[17,18]$, we proposed another FL-based CAC system called FACS-P, which considered the following parameters for acceptance decision: user Speed $(S p)$, user Angle $(A n)$, Service request $(S r)$, Correction value $(C v)$, Required bandwidth $(R q)$, Counter state $(C s)$, Accept or Reject decision $(A / R)$, Differentiated service $(D s)$, Real Time Counter $(R T C)$, and Non Real Time Counter (NRTC). The structure of the FACS-P is shown in Fig. 2. In FACS-P, as the only priority parameter is considered the Service request $(S r)$.

\section{Proposed Fuzzy Admission Control System}

In our previous work, we found that when using only one parameter for the priority, the FCAC-P system did not differentiate well different services. For this reason, in the new implemented system, we consider different priorities during the CAC decision.

The Fuzzy Logic Controller (FLC) is the main part of the proposed Fuzzy Admission Control System with Many

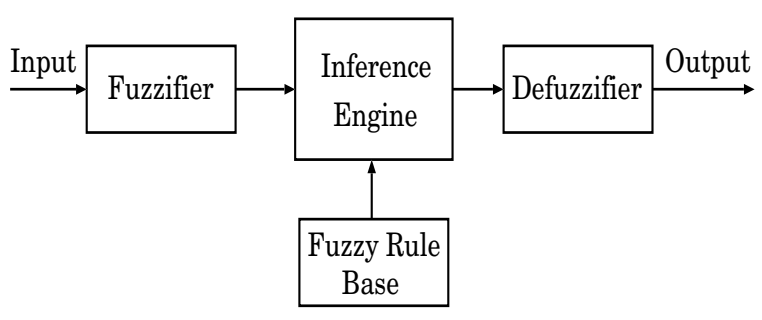

Figure 3. FLC structure.
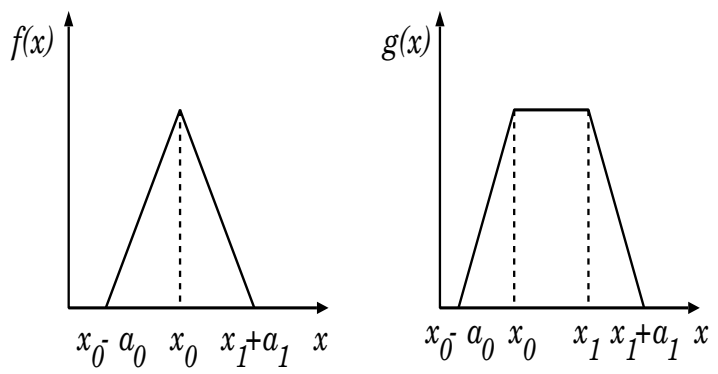

Figure 4. Triangular and trapezoidal membership functions.

Priorities (FACS-MP) and its basic elements are shown in Fig. 3. They are the fuzzifier, inference engine, Fuzzy Rule Base (FRB) and defuzzifier. As membership functions, we use triangular and trapezoidal membership functions because they are suitable for real-time operation [19-22]. The membership functions are shown in Fig. 4.

The proposed FACS-MP considers the following parameters for acceptance decision: user Speed $(S)$, user Angle $(A)$, Correction value $(C v)$, User priority $(U p)$, Service priority $(S p)$, Connection priority $(C p)$, Priority $(P)$, Counter state $(C s)$, Accept or Reject decision $(A / R)$, Differentiated service $(D s)$, Real Time Counter (RTC), and Non Real Time Counter (NRTC). The structure of the proposed FACS-MP is shown in Fig. 5.

\section{A. FLC1 Design}

The input parameters for FLC1 are: user Speed $(S)$ and user Angle $(A)$, while the output linguistic parameter is Correction value $(C v)$. The term sets of $S$, and $A$ are defined respectively as:

$$
\begin{aligned}
T(S)= & \{\text { Slow, Middle, Fast }\}=\{\text { Sl }, M, F\} \\
T(A)=\quad & \{\text { Back } 1, \text { Left } 1, \text { Left } 2, \text { Straight, Right } 1, \text { Right } 2, \\
& \text { Back } 2\}=\{B 1, \text { L1,L2,St,R } 1, R 2, B 2\} .
\end{aligned}
$$

The membership functions for input parameters of FLC1 are defined as follows:

$$
\begin{aligned}
\mu_{S l}(S) & =g\left(S ; S l_{0}, S l_{1}, S l_{w 0}, S l_{w 1}\right) \\
\mu_{M}(S) & =f\left(S ; M_{0}, M_{w 0}, M_{w 1}\right) \\
\mu_{F}(S) & =g\left(S ; F_{0}, F_{1}, F_{w 0}, F_{w 1}\right) \\
\mu_{B 1}(A) & =g\left(A ; B 1_{0}, B 1_{1}, B 1_{w 0}, B 1_{w 1}\right)
\end{aligned}
$$




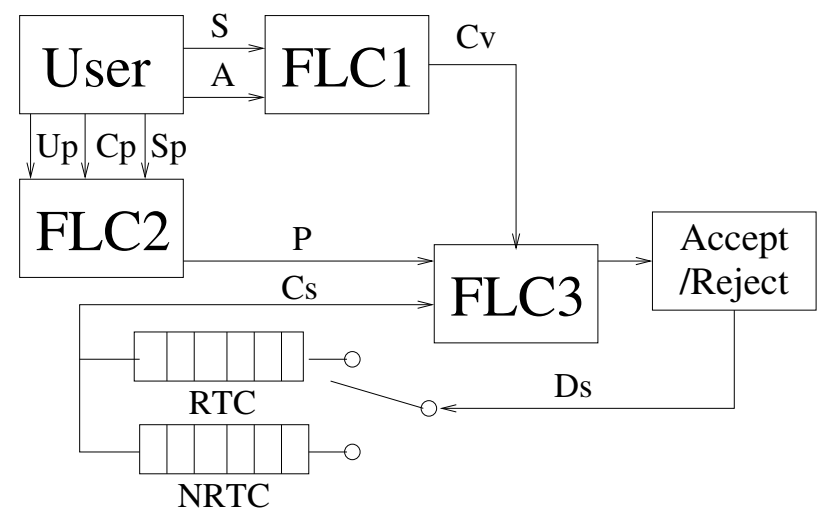

Figure 5. FACS-MP model.

$$
\begin{aligned}
\mu_{L 1}(A) & =f\left(A ; L 1_{0}, L 1_{w 0}, L 1_{w 1}\right) \\
\mu_{L 2}(A) & =f\left(A ; L 2_{0}, L 2_{w 0}, L 2_{w 1}\right) ; \\
\mu_{S t}(A) & =f\left(A ; S t_{0}, S t_{w 0}, S t_{w 1}\right) ; \\
\mu_{R 1}(A) & =f\left(A ; R 1_{0}, R 1_{w 0}, R 1_{w 1}\right) ; \\
\mu_{R 2}(A) & =f\left(A ; R 2_{0}, R 2_{w 0}, R 2_{w 1}\right) ; \\
\mu_{B 2}(A) & =g\left(A ; B 2_{0}, B 2_{1}, B 2_{w 0}, B 2_{w 1}\right) .
\end{aligned}
$$

The small letters $w 0$ and $w 1$ mean left width and right width, respectively.

The term set of the output linguistic parameter $T(\mathrm{Cv})$ is defined as $\{$ Correction value 1, Correction value $2, \ldots$, Correction value 9$\}=\{C v 1, C v 2, \ldots, C v 9\}$. The membership functions for the output parameter $C v$ are defined as follows:

$$
\begin{aligned}
& \mu_{C v 1}(C v)=g\left(C v ; C v 1_{0}, C v 1_{1}, C v 1_{w 0}, C v 1_{w 1}\right) ; \\
& \mu_{C v 2}(C v)=f\left(C v ; C v 2_{0}, C v 2_{w 0}, C v 2_{w 1}\right) ; \\
& \mu_{C v 3}(C v)=f\left(C v ; C v 3_{0}, C v 3_{w 0}, C v 3_{w 1}\right) ; \\
& \mu_{C v 4}(C v)=f\left(C v ; C v 4_{0}, C v 4_{w 0}, C v 4_{w 1}\right) ; \\
& \mu_{C v 5}(C v)=f\left(C v ; C v 5_{0}, C v 5_{w 0}, C v 5_{w 1}\right) ; \\
& \mu_{C v 6}(C v)=f\left(C v ; C v 6_{0}, C v 6_{w 0}, C v 6_{w 1}\right) ; \\
& \mu_{C v 7}(C v)=f\left(C v ; C v 7_{0}, C v 7_{w 0}, C v 7_{w 1}\right) ; \\
& \mu_{C v 8}(C v)=f\left(C v ; C v 8_{0}, C v 8_{w 0}, C v 8_{w 1}\right) ; \\
& \mu_{C v 9}(C v)=g\left(C v ; C v 9_{0}, C v 9_{1}, C v 9_{w 0}, C v 9_{w 1}\right) .
\end{aligned}
$$

The membership functions of FLC1 are shown in Fig. 6. The FRB forms a fuzzy set of dimensions $|T(S)| \times|T(A)|$, where $|T(x)|$ is the number of terms on $T(x)$. The FRB1 shown in Table 1 has 21 rules. The control rules have the following form: IF "conditions" THEN "control action".

\section{B. FLC2 Design}

The input parameters for FLC2 are: User priority $(U p)$, Connection priority $(C p)$, and Service priority $(S p)$, while the output linguistic parameter is Priority $(P)$. The term sets of $U p, C p$, and $S p$ are defined respectively as:
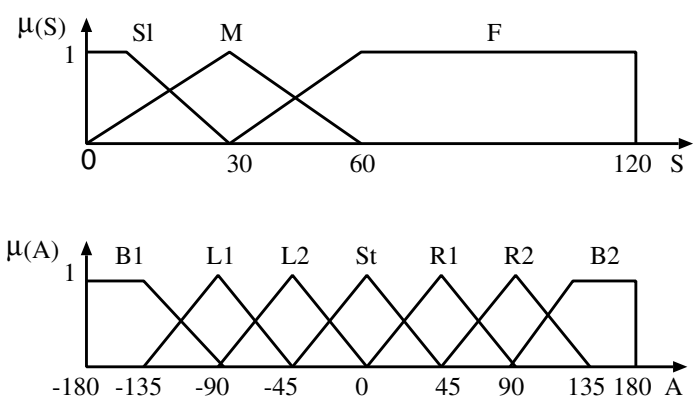

$\mu(\mathrm{Cv}) \uparrow_{\mathrm{Cv} 1} \mathrm{Cv} 2 \quad \mathrm{Cv} 3 \quad \mathrm{Cv} 4 \quad \mathrm{Cv} 5 \quad \mathrm{Cv} 6 \quad \mathrm{Cv} 7 \quad \mathrm{Cv} 8 \quad \mathrm{Cv} 9$

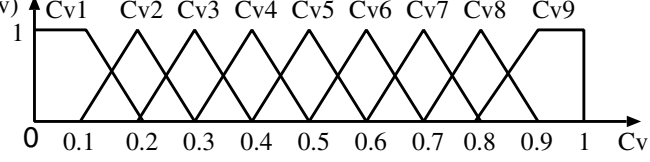

Figure 6. FLC1 membership functions.

Table I

FRB 1.

\begin{tabular}{|l|l|l|l|}
\hline \hline Rule & S & A & Cv \\
\hline 1 & S1 & B1 & Cv2 \\
2 & S1 & L1 & Cv3 \\
3 & S1 & L2 & Cv4 \\
4 & S1 & S & Cv7 \\
5 & S1 & R1 & Cv4 \\
6 & S1 & R2 & Cv3 \\
7 & S1 & B2 & Cv2 \\
8 & M & B1 & Cv1 \\
9 & M & L1 & Cv3 \\
10 & M & L2 & Cv3 \\
11 & M & St & Cv9 \\
12 & M & R1 & Cv3 \\
13 & M & R2 & Cv3 \\
14 & M & B2 & Cv1 \\
15 & Fa & B1 & Cv1 \\
16 & Fa & L1 & Cv2 \\
17 & Fa & L2 & Cv3 \\
18 & Fa & S & Cv9 \\
19 & Fa & R1 & Cv3 \\
20 & Fa & R2 & Cv2 \\
21 & Fa & B2 & Cv1 \\
\hline
\end{tabular}

$$
\begin{aligned}
T(U p) & =\{\text { Low priority, Middle priority, High priority }\} \\
& =\{\text { Lp, Mp, Hp }\} \\
T(C p) & =\{\text { Short }, \text { Middle, } \text { Long }\}=\{S h, M i, \text { Lo }\} \\
T(S p) & =\{\text { Text, Voice, }, \text { Video }\}=\{T, \text { Vo, Vi }\}
\end{aligned}
$$

The term set of the output linguistic parameter $T(P)$ is defined as $\{$ Priority 1, Priority $2, \ldots$, Priority 9$\}=\{P 1$, $P 2, \ldots, P 9\}$. The membership functions for the output parameter $P$ are defined as follows:

$$
\begin{aligned}
& \mu_{P 1}(P)=g\left(P ; P 1_{0}, P 1_{1}, P 1_{w 0}, P 1_{w 1}\right) \\
& \mu_{P 2}(P)=f\left(P ; P 2_{0}, P 2_{w 0}, P 2_{w 1}\right)
\end{aligned}
$$



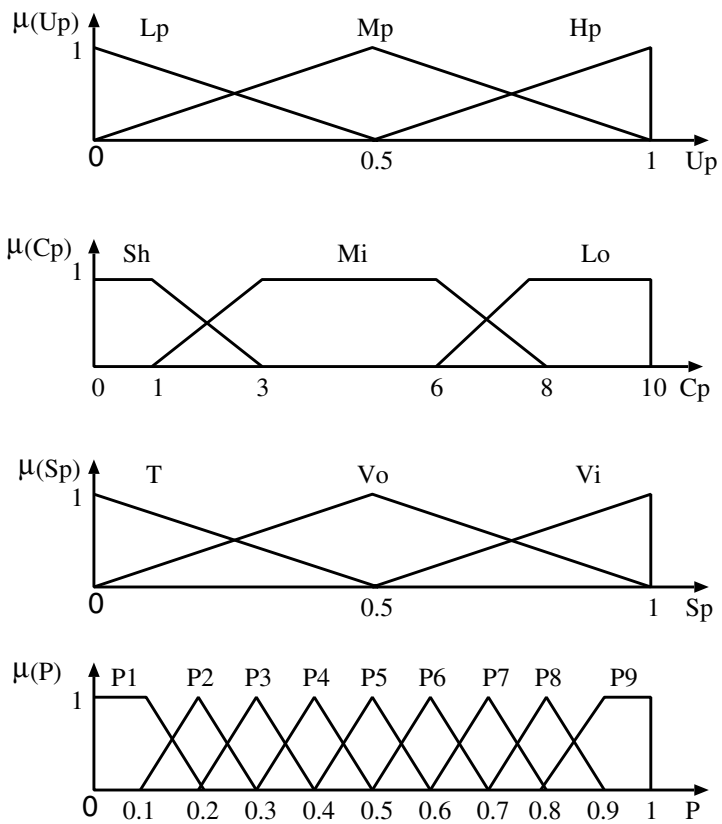

Figure 7. FLC2 membership functions.

$$
\begin{aligned}
& \mu_{P 3}(P)=f\left(P ; P 3_{0}, P 3_{w 0}, P 3_{w 1}\right) ; \\
& \mu_{P 4}(P)=f\left(P ; P 4_{0}, P 4_{w 0}, P 4_{w 1}\right) ; \\
& \mu_{P 5}(P)=f\left(P ; P 5_{0}, P 5_{w 0}, P 5_{w 1}\right) ; \\
& \mu_{P 6}(P)=f\left(P ; P 6_{0}, P 6_{w 0}, P 6_{w 1}\right) ; \\
& \mu_{P 7}(P)=f\left(P ; P 7_{0}, P 7_{w 0}, P 7_{w 1}\right) ; \\
& \mu_{P 8}(P)=f\left(P ; P 8_{0}, P 8_{w 0}, P 8_{w 1}\right) ; \\
& \mu_{P 9}(P)=g\left(P ; P 9_{0}, P 9_{1}, P 9_{w 0}, P 9_{w 1}\right) .
\end{aligned}
$$

The membership functions of FLC2 are shown in Fig. 7. The FRB2 shown in Table 2 has 27 rules.

\section{FLC3 Design}

For the design of FLC3, we keep the same parameters with our previous work. The input parameters for FLC3 are: the output parameter of the FLC1 $(C v)$, Priority $(P)$, and the Counter state $(C s)$, which shows the capacity of the system. While, the output linguistic parameter is the Accept/Reject decision $(A / R)$.

The term sets of $C v, P$, and $C s$ are defined as:

$$
\begin{aligned}
T(C v) & =\{\text { Bad, Normal, Good }\}=\{B, N, G\} \\
T(P) & =\{\text { Low priority, Middle priority, High priority }\} \\
& =\{\text { Lwp, Mdp, Hgp }\} ; \\
T(C s) & =\{\text { Small, Middle, Full }\}=\{\text { Sm, Md, Fu }\} .
\end{aligned}
$$

In order to have a soft admission decision, for the output linguistic parameter $(A / R)$, we considered not only "accept" and "reject" but also "weak accept", "weak reject", and "not accept not reject" for the accept/reject decision. The membership functions for input and output linguistic parameters
Table II

FRB2

\begin{tabular}{|l|l|l|l|l|}
\hline \hline Rule & Up & Cp & Sp & P \\
\hline 1 & Lp & Sh & T & P6 \\
2 & Lp & Sh & Vo & P5 \\
3 & Lp & Sh & Vi & P4 \\
4 & Lp & Mi & T & P5 \\
5 & Lp & Mi & Vo & P3 \\
6 & Lp & Mi & Vi & P2 \\
7 & Lp & Lo & T & P4 \\
8 & Lp & Lo & Vo & P2 \\
9 & Lp & Lo & Vi & P1 \\
10 & Mp & Sh & T & P5 \\
11 & Mp & Sh & Vo & P6 \\
12 & Mp & Sh & Vi & P5 \\
13 & Mp & Mi & T & P4 \\
14 & Mp & Mi & Vo & P5 \\
15 & Mp & Mi & Vi & P4 \\
16 & Mp & Lo & T & P3 \\
17 & Mp & Lo & Vo & P4 \\
18 & Mp & Lo & Vi & P3 \\
19 & Hp & Sh & T & P8 \\
20 & Hp & Sh & Vo & P9 \\
21 & Hp & Sh & Vi & P9 \\
22 & Hp & Mi & T & P7 \\
23 & Hp & Mi & Vo & P8 \\
24 & Hp & Mi & Vi & P9 \\
25 & Hp & Lo & T & P6 \\
26 & Hp & Lo & Vo & P7 \\
27 & Hp & Lo & Vi & P8 \\
\hline
\end{tabular}

of FLC3 are shown in Fig. 8. The FRB3 shown in Table 3 has 27 rules.

The membership functions for input parameters of FLC3 are defined as follows:

$$
\begin{aligned}
\mu_{B}(C v) & =f\left(C v ; B_{0}, B_{w 0}, B_{w 1}\right) ; \\
\mu_{N}(C v) & =f\left(C v ; N_{0}, N_{w 0}, N_{w 1}\right) ; \\
\mu_{G}(C v) & =f\left(C v, G_{0}, G_{w 0}, G_{w 1}\right) ; \\
\mu_{L w p}(P) & =f\left(P ; L w p_{0}, L w p_{w 0}, L w p_{w 1}\right) ; \\
\mu_{M d p}(P) & =f\left(P ; M d p_{0}, M d p_{w 0}, M d p_{w 1}\right) ; \\
\mu_{H g p}(P) & =f\left(P ; H g p_{0}, H g p_{w 0}, H g p_{w 1}\right) ; \\
\mu_{S m}(C s) & =f\left(C s ; S m_{0}, S m_{w 0}, S m_{w 1}\right) ; \\
\mu_{M d}(C s) & =f\left(C s ; M d_{0}, M d_{w 0}, M d_{w 1}\right) ; \\
\mu_{F u}(C s) & =f\left(C s ; F u_{0}, F u_{w 0}, F u_{w 1}\right) .
\end{aligned}
$$

The term set of the output linguistic parameter $T(A / R)$ is defined as $\{$ Reject, Weak Reject, Not Reject Not Accept, Weak Accept, Accept $\}$. We write for short as $\{R$, WR, NRNA, WA, A $\}$. The membership functions for the output parameter $A / R$ are defined as follows:

$$
\begin{aligned}
\mu_{R}(A / R) & =g\left(A / R ; R_{0}, R_{1}, R_{w 0}, R_{w 1}\right) ; \\
\mu_{W R}(A / R) & =f\left(A / R ; W R_{0}, W R_{w 0}, W R_{w 1}\right) ; \\
\mu_{N R N A}(A / R) & =f\left(A / R ; N R N A_{0}, N R N A_{w 0}, N R N A_{w 1}\right) ; \\
\mu_{W A}(A / R) & =f\left(A / R ; W A_{0}, W A_{w 0}, W A_{w 1}\right) ; \\
\mu_{A}(A / R) & =g\left(A / R ; A_{0}, A_{1}, A_{w 0}, A_{w 1}\right) .
\end{aligned}
$$




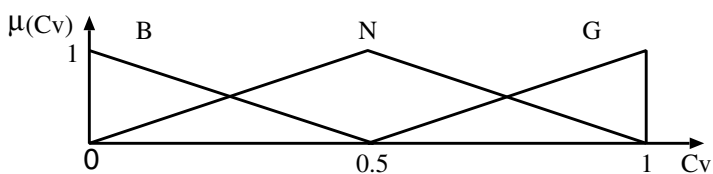

$\mu(\mathrm{P})$

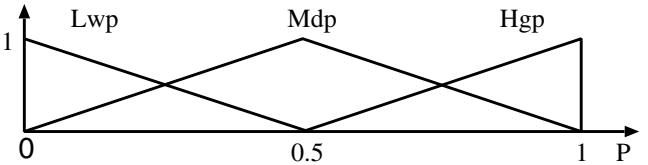

$\mu(\mathrm{Cs}) \uparrow \quad \mathrm{Sm} \quad \mathrm{Md} \quad \mathrm{Fu}$
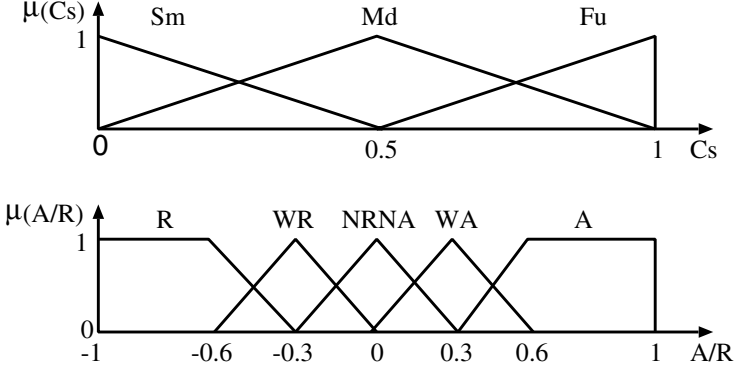

Figure 8. FLC3 membership functions.

Table III

FRB3.

\begin{tabular}{|l|l|l|l|l|}
\hline \hline Rule & Cv & P & Cs & A/B \\
\hline 1 & B & Lwp & Sm & WA \\
2 & B & Lwp & Md & NRNA \\
3 & B & Lwp & Fu & WR \\
4 & B & Mdp & Sm & A \\
5 & B & Mdp & Md & NRNA \\
6 & B & Mdp & Fu & WR \\
7 & B & Hgp & Sm & A \\
8 & B & Hgp & Md & NRNA \\
9 & B & Hgp & Fu & NRNA \\
10 & N & Lwp & Sm & WA \\
11 & N & Lwp & Md & NRNA \\
12 & N & Lwp & Fu & NRMA \\
13 & N & Mdp & Sm & A \\
14 & N & Mdp & Md & NRNA \\
15 & N & Mdp & Fu & NRNA \\
16 & N & Hgp & Sm & A \\
17 & N & Hgp & Md & NRNA \\
18 & N & Hgp & Fu & NRNA \\
19 & G & Lwp & Sm & A \\
20 & G & Lwp & Md & A \\
21 & G & Lwp & Fu & R \\
22 & G & Mdp & Sm & A \\
23 & G & Mdp & Md & A \\
24 & G & Mdp & Fu & WR \\
25 & G & Hgp & Sm & A \\
26 & G & Hgp & Md & A \\
27 & G & Hgp & Fu & NRNA \\
\hline & & & & \\
\hline
\end{tabular}

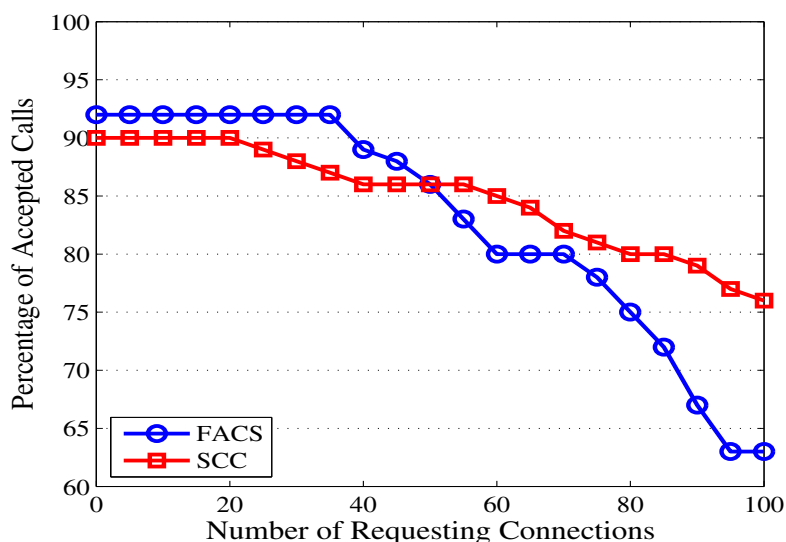

Figure 9. Comparison of FACS with SCC.

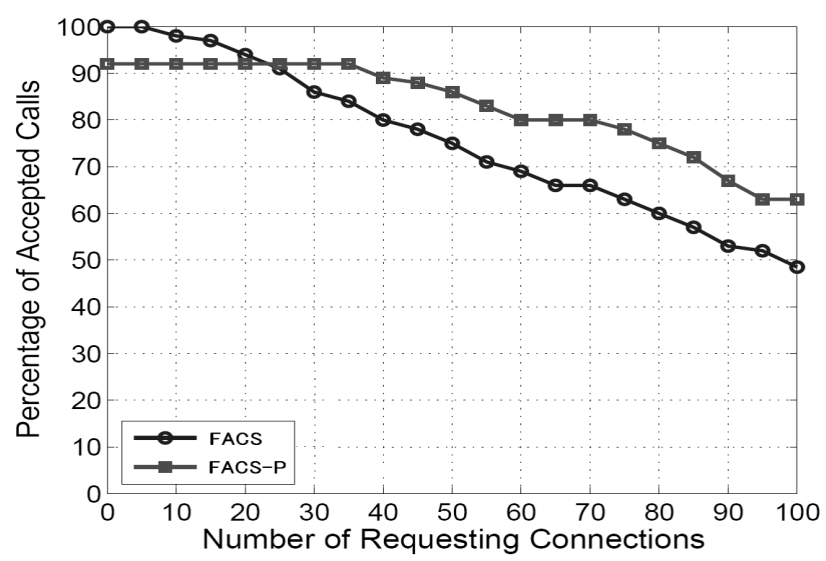

Figure 10. Comparison of FACS-P with FACS

\section{Simulation Results}

For simulations, we used FuzzyC simulator implemented in our lab. The FuzzyC is implemented by $\mathrm{C}$ language. The simulation were carried out in Linux Ubuntu 9.10 computer.

In order to make a fair comparison between the proposed system and previous systems, we considered the following parameters for simulations:

- the user speed was from 0 to $120 \mathrm{~km} / \mathrm{h}$,

- the user direction was changed from -180 degree to +180 degree,

- the requested size was 1,5 and 10 Bandwidth Units (BU) for text, voice and video, respectively,

- the bandwidth of the BS was considered $40 \mathrm{BU}$.

In Fig. 9, we show the performance of FACS and SCC. When the number of requesting connections is less than 50 , the percentage of accepted calls for proposed system is higher than SCC. However, when the number of requesting connections is larger than 50 , the proposed system accepts less number of connections. This is because, the proposed system guarantees the QoS of ongoing calls and requesting 


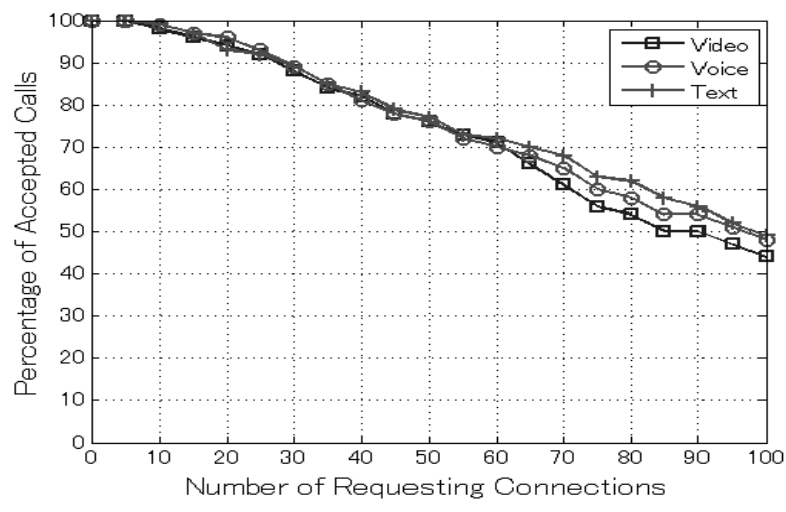

Figure 11. Performance of FACS-P for different services.

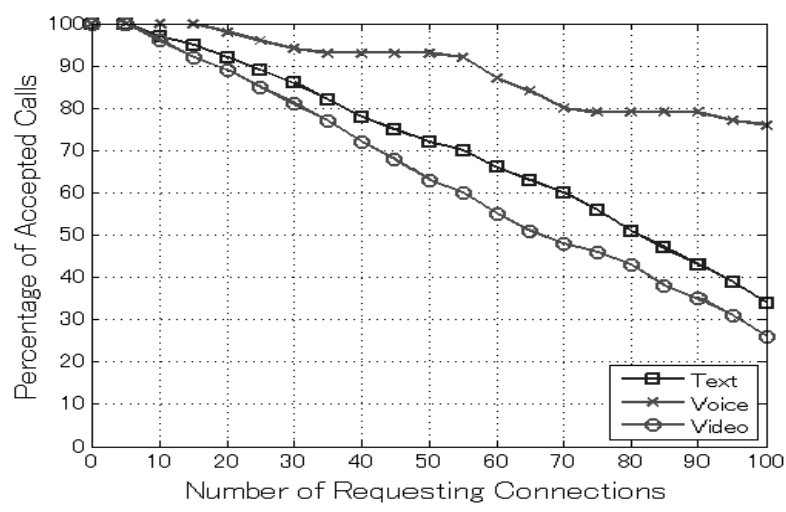

Figure 12. Performance of FACS-MP for different services.

connections. When the number of requesting connections is less than 50 (the case when there is enough $\mathrm{BU}$ ), the proposed system make a better allocation of the resources compared with SCC.

In Fig. 10, we show the performance of FACS and FACS$\mathrm{P}$. When the number of requesting connections is less than 25, the percentage of accepted calls for FACS system is higher than FACS-P system. However, when the number of requesting connections is larger than 25, the FACS-P system accepts less number of connections. This is because, the FACS-P system guarantees the QoS of on-going calls, thus decreasing the acceptance ratio of the requesting connections. However, by using only one priority parameter, the FACS-P system can not differentiate well different services as shown in Fig. 11.

In Fig. 12 and Fig. 13, we show the performance of FACSMP. As can be seen by this figure, the proposed system shows a good behaviour, by making a good differentiation for different services and different priorities.

In Fig. 14, we show the performance comparison between FACS-MP and FACS-P. When the number of requesting connections is small, the percentage of accepted calls for FACS-P and FACS-MP is almost the same. However, when

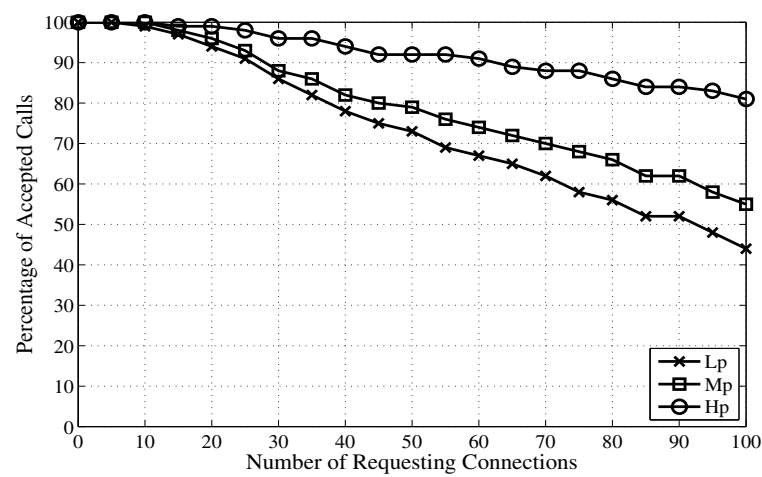

Figure 13. Performance of FACS-MP for different priorities.

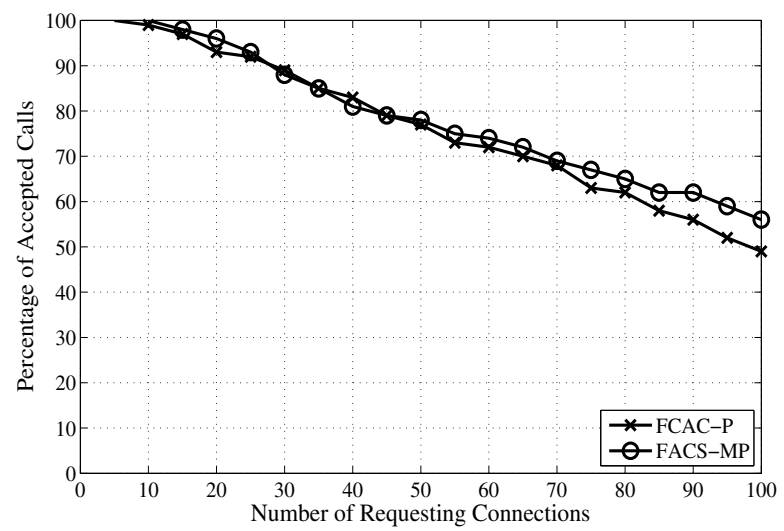

Figure 14. Performance comparison between FACS-MP and FACS-P.

the number of requesting connections is larger than 45 , the FACS-MP system accepts more number of connections.

\section{CONCLUSIONS}

In this paper, we proposed a FL-based CAC system with different priorities called FACS-MP. We evaluated and compared the performance of different fuzzy-based CAC systems.

We evaluated the performance of the proposed FACSMP system for different services and priorities. From the simulations results, we conclude that the proposed FACSMP make a good differentiation for different services and priorities.

In the future, we would like to evaluate the proposed FACS-MP by extensive simulations.

\section{REFERENCES}

[1] Y. Fang, and Y. Zhang, "Call Admission Control Schemes and Performance Analysis in Wireless Mobile Networks", IEEE Trans. on Vehicular Technology, Vol. 51, No. 2, pp. 371-382, March 2002. 
[2] W. Wang, X. Wang, and A. A. Nilsson, "Energy-Efficient Bandwidth Allocation in Wireless Networks: Algorithms, Analysis, and Simulations", IEEE Transactions on Wireless Communications, Vol 5, No. 5, pp. 1103-1114, May 2006.

[3] D. Z. Deniz and N. O. Mohamed, "Performance of CAC Strategies for Multimedia Traffic in Wireless Networks", IEEE Journal on Selected Areas in Commun., Vol. 21, No. 10, pp. 1557-1565, December 2003.

[4] L. Barolli, A. Koyama, T. Yamada, S. Yokoyama, T. Suganuma, N.Shiratori, "A Fuzzy Admission Control Scheme and Its Performance Evaluation", IPSJ Journal, Vol. 42, No. 12, pp. 3213-3221, December 2001.

[5] L. Barolli, A. Koyama, T. Suganuma and N. Shiratori, "A Genetic Algorithm Based QoS Routing Method for Multimedia Communications Over High-Speed Networks", IPSJ Journal, Vol. 44, No. 2, pp. 544-552, February 2003.

[6] B. Bensaou, S. T. C. Lam, H-W. Chu, D. H. K. Tsang, "Estimation of the Cell Loss Ratio in ATM Networks with a Fuzzy System and Application to Measurement-Based Call Admission Control", IEEE/ACM Trans. on Networking, Vol. 5, No. 4, pp. 572-584, August 1997.

[7] I. Habib, (Ed.), "Neurocomputing in High-Speed Networks", IEEE Commun. Magazine, Special Issue, Vol. 33, No. 10, 1995.

[8] L. Barolli, A. Koyama, T. Suganuma, N. Shiratori, "GAMAN: A GA Based QoS Routing Method for Mobile Ad-hoc Networks", Journal of Interconnection Networks (JOIN), Vol. 4, No. 3, pp. 251-270, September 2003.

[9] A. Koyama, L. Barolli, G. Capi, B. O. Apduhan, J. Arai, A.Durresi, "An Efficient Multi-Purpose Optimization Method for QoS Routing Using Genetic Algorithm", Journal of Interconnection Networks (JOIN), Vol. 5, No. 4, pp. 409-428, December 2004.

[10] M. Ikeda, L. Barolli, A. Koyama, A. Durresi, G. De Marco, J. Iwashige, "Performance Evaluation of an Intelligent CAC and Routing Framework for Multimedia Applications in Broadband Networks", Journal of Computer and System Science (JCSS), Vol. 72, Issue 7, pp. 1183-1200, November 2006.

[11] L. Barolli, M. Ikeda, G. De Marco, A. Durresi, A. Koyama, J. Iwashige, "A Search Space Reduction Algorithm for Improving the Performance of a GA-based Routing Method in Ad-Hoc Network", International Journal of Distributed Sensor Networks (IJDSN), Vol. 3, No. 1, pp. 41-57, January 2007.

[12] L. Barolli, "A Speed-Aware Handover System for Wireless Cellular Networks Based on Fuzzy Logic", Mobile Information Systems (MIS), Vol. 4, No. 1, pp. 1-12, 2008.

[13] L. Barolli, J. Anno, F. Xhafa, A. Durresi, A. Koyama, "A Context-Aware Fuzzy-Based Handover System for Wireless Cellular Networks and Its Performance Evaluation", Journal of Mobile Multimedia (JMM), Vol. 4. No. 3/4, pp. 241-258, October 2008.

[14] L. Barolli, F. Xhafa, A. Durresi, A. Koyama, ”A Fuzzy-based Call Admission Control System for Wireless Cellular Networks", Proc. of MNSA-2007/ICDCS-2007, Toronto, Canada, CD-ROM, 8 pages, June 2007.
[15] L. Barolli, "An Intelligent Call Admission Control System for Wireless Cellular Networks Based on Fuzzy Logic", Journal of Mobile Multimedia (JMM), Vol. 3. No. 4, pp. 331-346, December 2007.

[16] D. A. Levine, I. F. Akyildiz, M. Naghshineh, "A Resource Estimation and Call Admission Algorithm for Shadow Cluster Concept", IEEE/ACM Transactions on Networking, Vol. 5, No. 1, pp. 1-12, 1997.

[17] Gj. Mino, L. Barolli, A. Durresi, F. Xhafa, A. Koyama, "A Fuzzy-based Call Admission Control Scheme for Wireless Cellular Networks Considering Priority of On-going Connections", Proc. of ICDCS-2009 Workshops, MNSA-2009, Montreal, Canada, pp. 380-387, June 2009.

[18] Gj. Mino, L. Barolli, F. Xhafa, A. Durresi, A. Koyama, "Performance Evaluation of a Fuzzy-based CAC Scheme for Wireless Cellular Networks: A Case Study Considering Priority of On-going Connections", International Journal of Business Intelligence and Data Mining, Vol. 5, No. 3, pp. 269$284,2010$.

[19] D. Dubois, H. Prade, R. Yager, (Eds.), "Fuzzy Sets for Intelligent Systems", Morgan Kaufman Publishers, 1993.

[20] L. A. Zadeh, "Fuzzy Logic, Neural Networks, and Soft Computing", Communication of ACM, Vol. 37, No. 3, pp. 7784, March 1994.

[21] R. R. Yager, and L. A. Zadeh (Editors), "An Introduction To Fuzzy Logic Applications In Intelligent Systems", Kluwer Academic Publishers, 1992.

[22] H. J. Zimmermann, "Fuzzy Set Theory-and Its Applications", Kluwer Academic Publishers, Second Revised Edition, 1991. 